\section{Low Energy, Low Angle, Large Area Ion Polishing for Improved EBSD Indexing}

S.D. Walck*, J.R. Porter ${ }^{\star *}$, H-W. Yang ${ }^{\star *}$, S.S. Dheda ${ }^{* * *}$

${ }^{*}$ South Bay Technology, Inc., San Clemente, CA

${ }^{*}$ California Institute for Telecommunications and Information Technology, UC Irvine, CA

${ }^{* *}$ Dept. of Chemical Engineering and Materials Science, UC Irvine, CA swalck@southbaytech.com

Good sample preparation is essential for acquiring successful electron backscattered diffraction (EBSD) patterns in the SEM. Mechanical polishing to obtain the required surface quality with minimal sub-surface defects and deformation that does not interfere with the quality of the diffraction data is, more often than not, an art form. Special polishing techniques, such as low force lapping fixtures, electrochemical-mechanical polishing, and vibratory polishing, have been used to minimize the sub-surface damage, but have not eliminated it. Ion polishing has been used to reduce the damage layer further. However, the commercially available ion systems suffer several drawbacks, including: 1 ) small area treatment ( $\leq$ $1 \mathrm{~cm})$ 2) decreasing beam current density with accelerating voltages, and 3) the inability to process non-conducting samples. Barna and Pecz have shown that at $3 \mathrm{keV}$ with an incident angle of $5^{\circ}$ relative to the surface, approximately $25 \mathrm{~nm}$ of ion damage occurs in $\mathrm{Si}$ and $\mathrm{GaAs}$, but at $250 \mathrm{eV}$, there is less than $1 \mathrm{~nm}$ of amorphization of the surface [1]. They also showed that a glancing angle across the surface is essential for removing topographic features [2]. The ion guns that have been available for ion polishing and ion etching of SEM samples typically cannot operate effectively below $3 \mathrm{keV}$ because of the low current density.

A Kaufman-type $1 \mathrm{~cm}$ DC ion source (KDC-10) was adapted to replace the standard ion gun in the etch port of a South Bay Technology, Inc. sputter coating/etching system (Model IBS/e). Figure 1 shows the ion source in position in the IBS/e. The source to center of sample distance is $5.8 \mathrm{~cm}$. Figure 2 shows the IBS/e with the power supply for the ion source together with a mass flow controller to measure the gas flow. This ion source overcomes the above limitations of the high voltage, focusing ion guns. It has a $1 \mathrm{~cm}$ beam size that can be collimated, convergent, or divergent. These different conditions are achieved by changing the carbon grid geometry that

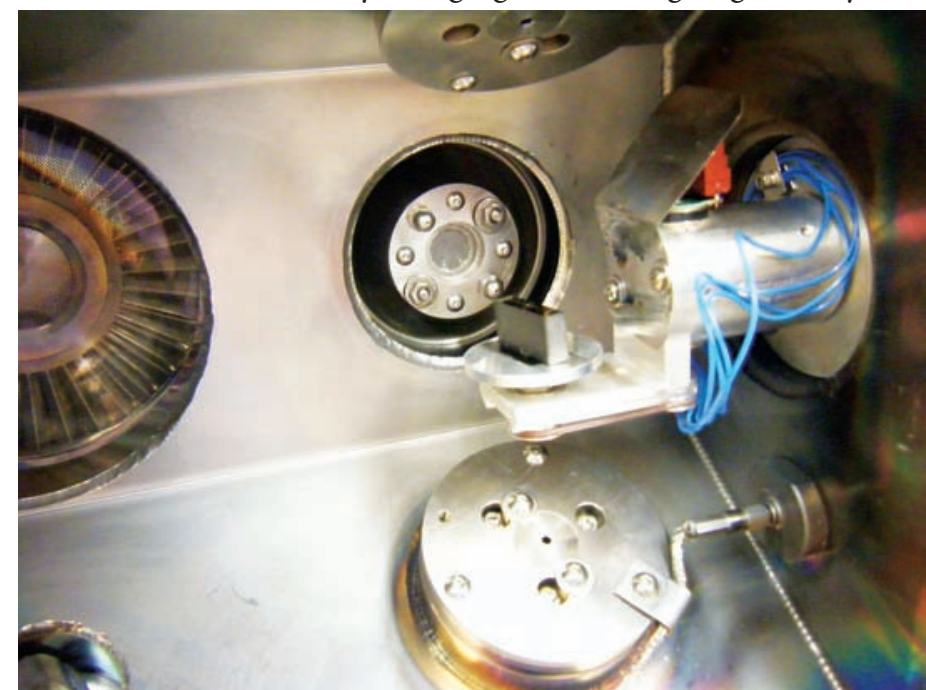

Figure $1 \mathrm{KDC}-101 \mathrm{~cm}$ kaufman Ion source in the etch port of the SBT IBS/e.

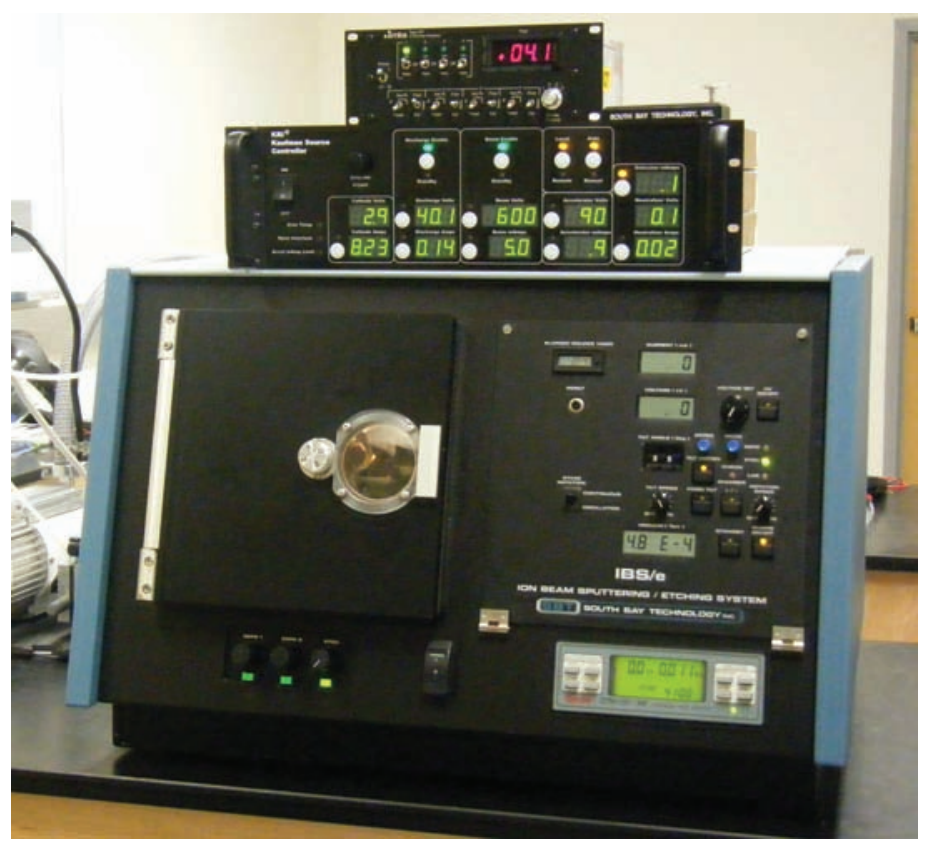

Figure 2 Model IBS/e equipped with the KDC-10 ion source. The ion source power supply is on top and a MKS mass flow controller was used to set up the gas flow conditions for the system.

\section{Table 1}

Maximum Ar Ion Beam Current for $1 \mathrm{~cm}$ Ion Source

\begin{tabular}{|c|c|}
\hline Beam Voltage $(\mathrm{V})$ & Beam Current $(\mathrm{mA})$ \\
\hline 100 & 1 \\
\hline 200 & 2 \\
\hline 300 & 4 \\
\hline 400 & 7 \\
\hline 500 & 11 \\
\hline 600 & 14 \\
\hline 700 & 20 \\
\hline 800 & 26 \\
\hline 900 & 32 \\
\hline 1000 & 38 \\
\hline
\end{tabular}

is used to extract and form the beam. It can be operated between $100 \mathrm{eV}$ and $1200 \mathrm{eV}$ with a high beam current. A neutralizer filament can be used to produce a neutral beam of gas ions that can ion process non-conducting samples. Table 1 shows the maximum current that the KDC-10 can produce at various energy settings. A typical ion beam profile is very close to a Gaussian distribution. For the KDC-10 operating at $600 \mathrm{~V}$ and a ion current of $10 \mathrm{~mA}$ with focusing grids ( $5 \mathrm{~cm}$ focal length), the maximum ion current density is $20 \mathrm{~mA} / \mathrm{cm}^{2}$ with a FWHM beam diameter value of about $7 \mathrm{~mm}$. With the collimating grids, the FWHM value is about $8 \mathrm{~mm}$. For both distributions, the ion current density falls below $0.2 \mathrm{~mA} /$ $\mathrm{cm}^{2}$ at distances greater than $10 \mathrm{~mm}$ from the center of the beam. The KDC-10, operating with the collimating grids, has a minimum half-angle of about 5 degrees. Figure 3 shows a photograph of two pennies that were mounted on 2 inch diameter specimen carriers 


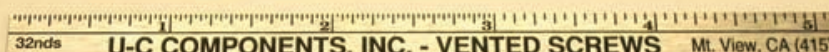

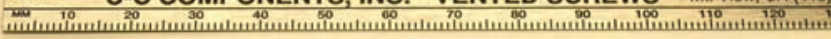

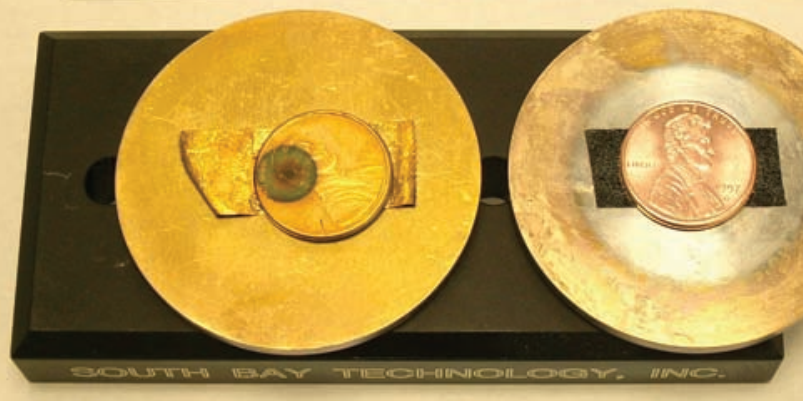

Figure 3 Photograph of two samples Ar ion treated at normal incidence with the standard ion gun (left) and the KDC-10 (right) with after they were heavily coated with gold.

for the IBS/e, coated with a heavy layer of gold, and then hit with the Ar ion beam from the standard ion gun (left side) and with the KDC-10 (right side) using normal incidence. The ion gun for the sample on the left was used at $8 \mathrm{kV}$ at an ion emission of $3 \mathrm{~mA}$. For the sample on the right, the KDC-10 was operated at $600 \mathrm{~V}$ and $5 \mathrm{~mA}$. Incidentally, the penny on the right was tarnished before coating and the ion processing cleaned that as well.

This article reports on the initial results of using the KDC-10 in the IBS/e to ion polish samples for EBSD analysis. To demonstrate the use of the KDC-10, a number of copper samples were tested. These included copper vias in silicon samples, copper layers on integrated circuit package substrates, and OFHC copper rods. Being soft metal with a tendency to smear when mechanically polished, these samples offer a significant challenge to EBSD analysis. The very first sample treated with the KDC-10 for an EBSD sample showed a dramatic improvement in the pattern quality compared to a mechanically polished sample. Figures $4 \mathrm{~A}$ and $4 \mathrm{~B}$ show the EBSD pattern of copper from an integrated circuit package substrate after a mechanical polish with $0.3 \mu \mathrm{m} \mathrm{Al}_{2} \mathrm{O}_{3}$ suspension followed by a short $0.05 \mu \mathrm{m}$ silica polish. No special sample preparation procedures were taken for these samples. They were mounted in an acrylic mounting block and hand polished with conventional lapping
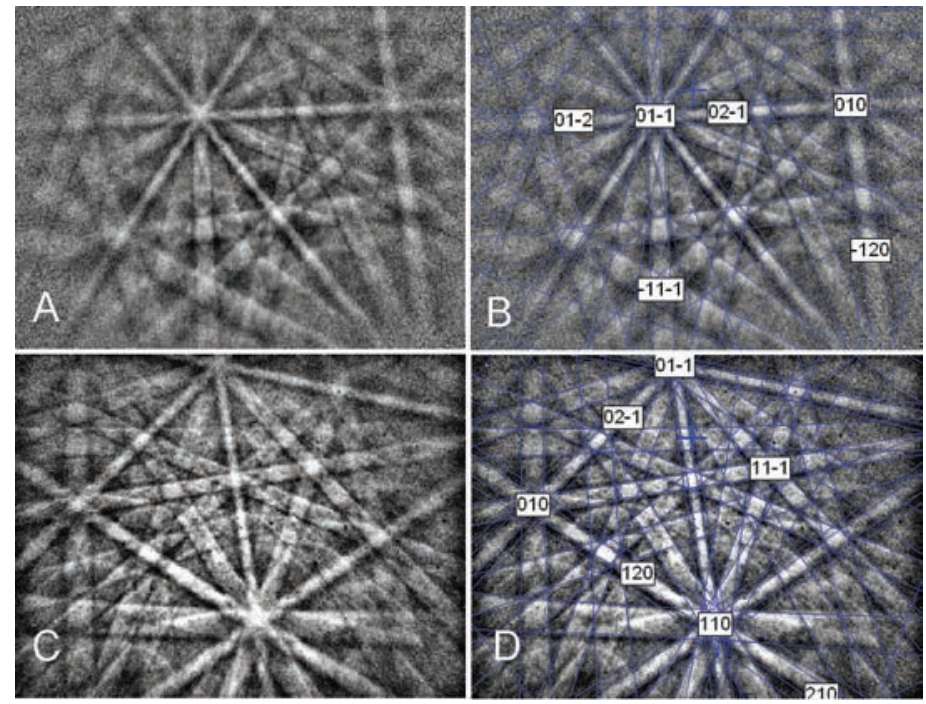

Figure 4. Indexed pattern of copper before ( $A$ and $B)$ and after $(C$ and D) ion polish. (B and D are indexed.) apparatus. A pattern from the same sample and as close to the same area as possible is shown in Fig. 4C and 4D after Ar ion polishing with the KDC-10 using the collimating grids and beam energy of $250 \mathrm{eV}$ for $40 \mathrm{~min}$ at an incidence angle of $5^{\circ}$ and a beam current of $2.5 \mathrm{~mA}$. Immediately obvious during the collection of the map was the significant increase in contrast of all of the patterns in the map compared to the contrast in the patterns for the mechanically polished samples. Figure 5 shows a band contrast map and a Z-oriented inverse pole figure colorization of a region of the copper within the printed circuit board. The ion pol-

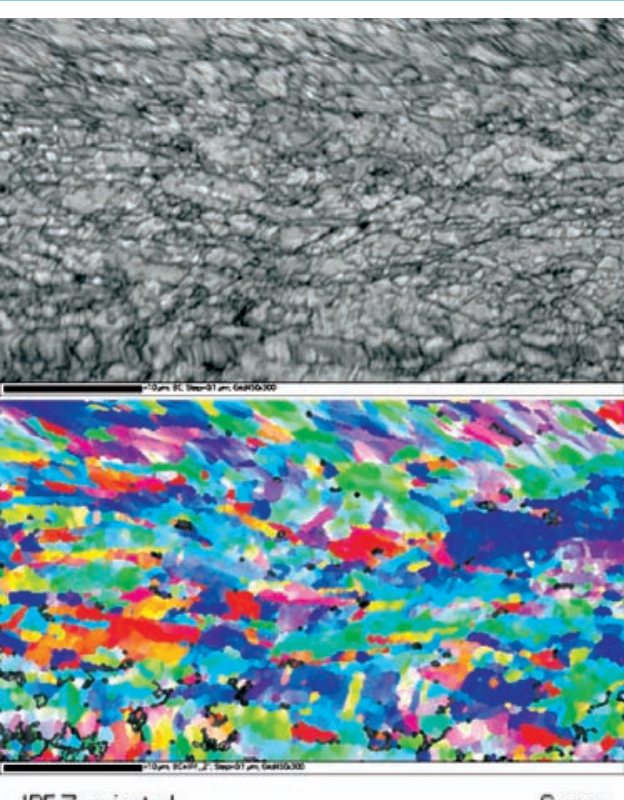

IPF-Z projected

21

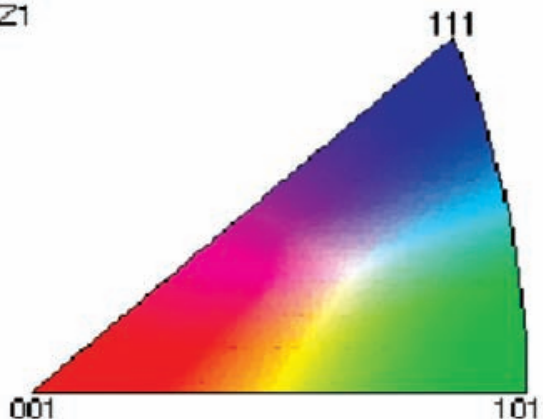

Figure 5. Band contrast image and Z-IPF colorization of copper cross-section in printed circuit board. Dynamic focusing correction was not available for this map.

ishing step improved the EBSD "hit rate" (fraction of pixels with successful indexing) from about $41 \%$ to $81 \%$ and that is attributed to a significant reduction in smearing due to the poor mechanical polishing. With some noise reduction, the software was readily able to identify boundaries, including CSL boundaries in the copper. It is interesting to note that for the mechanically polished sample, slight rotation of patterns taken from within a single grain were seen during the acquisition process, but in the ion polished sample the patterns were constant. This indicated the absence of the strain from polishing.

Although we saw a significant improvement in the "hit rate", this is not an appropriate measure for describing the change in the patterns due to sample preparation. This is because the "hit rate" is dependent on the grain size from sample to sample. Mixed patterns from a pattern collected on a grain boundary would not be able to be indexed and would be considered a "miss" in the software. What this means is that it would be very difficult to compare results from one sample to another. In order to better quantify the improvement from ion processing, Band Contrast maps were used to generate histograms from the EBSP (Electron Backscattered Pattern) quality numbers from each map collected. The maps generated were $70 \times$ 70 pixels in size, which gave histograms from 4900 patterns. This size was a balance between collection time and sufficient patterns to give sufficient statistics to generate the distributions. Since the 


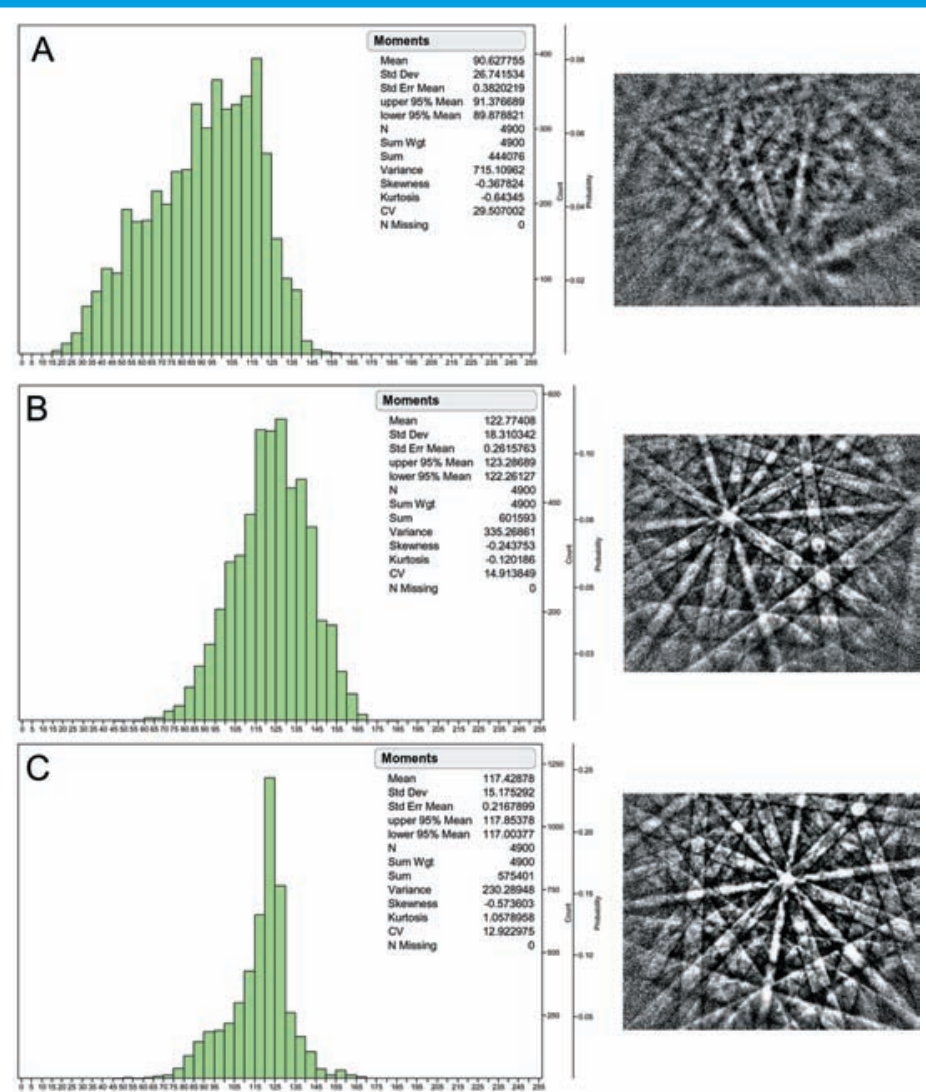

Figure 6 A) Colloidal silica polished copper via sample in silicon with a selected representative poorer quality pattern. B) ion polished and exposed to air for 2 days, C) Re-ion polished for $10 \mathrm{~m}$.

quality number for a pattern can be strongly influenced by the crystallographic orientation of the grain from which the pattern was acquired, the magnification and scanned areas were selected in order to generate enough randomness in the orientations to minimize the influence of large grains influencing the distribution. These histograms were collected from maps that were collected from 70 $\times 70$ pixel maps with sufficient different grains to insure variety in the orientations. For the different types of samples, the step size of the map was selected to maximize the number of different orientations, not to insure a good quality map. $4 \times 4$ binning was used for the maps. In the future, we would like to know whether the binning influences the EBSP quality number distributions. The initial EBSD mapping was done using an Oxford Instruments (HKL) Nordlys camera interfaced to a Zeiss LS15 EVO SEM and latter samples were examined with the same EBSD system on a Zeiss Ultra Plus SEM. Optimal conditions were used for each microscope. Because of the geometry of the detector position on the LS15 EVO relative to the stage, dynamic focusing correction was not available in that instrument.

The band contrast EBSP histograms clearly reflect the quality of the sample preparation. Figure 6A shows the Band Contrast EBSP quality histogram for a copper via in a silicon sample that has been polished with $0.05 \mu \mathrm{m}$ colloidal silica after it had been tripod polished and subsequently ion polished. The mapping process showed some good quality patterns as well as poor quality. That is reflected in the width and the position of the distribution towards lower values. The EBSP in Fig. 6A is representative of the poorer quality patterns. The better patterns are similar to those in the ion processed samples in Fig. $6 \mathrm{~B}$ and $6 \mathrm{C}$. Figure $6 \mathrm{~B}$ shows a sample that had been ion polished using $600 \mathrm{~V}$ and $5 \mathrm{~mA}$ at an angle of $5^{\circ}$ relative to the surface for 30 minutes, but had not been immediately imaged and was exposed to air for 2 days. The histogram has shifted to higher values and the distribution has narrowed, indicating the improvement of the quality of the patterns. This sample was then subjected to a 10 minute ion polish using the same ion processing parameters and immediately put into the microscope. Figure 6C shows that the mean of the distribution had not changed, but that the distribution had narrowed considerable. It strongly suggests that the difference in distribution is due to absence of the thin, native oxide layer on the surface.
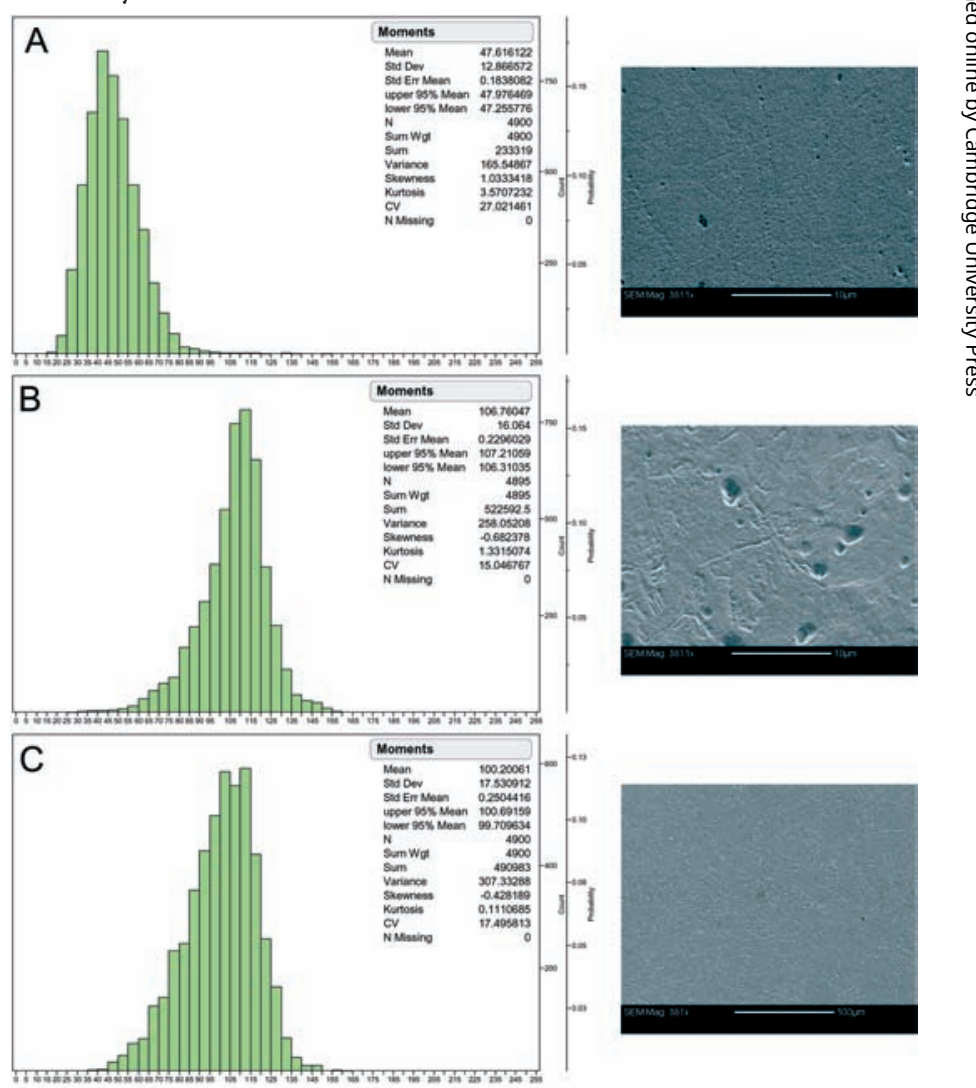

Figure 7 A) OFHC copper rod mechanically polished with $0.05 \mu \mathrm{m}$ colloidal silica and then heavily oxidized with an Ar/O2 plasma. The "hit" rate for this sample was about 10-12\%. B) Same sample as (A) after ion polishing for 13 minutes. The pattern was taken with a $0.3 \mu \mathrm{m}$ step size. C) Same sample as (B), but the pattern was taken with a $3.0 \mu \mathrm{m}$ step size.

Figure 7A shows the data from a polished OFHC copper rod that was mechanically polished with $0.05 \mu \mathrm{m}$ colloidal silica and then processed in a South Bay Technology PC-2000 plasma cleaner with an $\mathrm{Ar} / \mathrm{O}_{2}$ gas mixture to form a thicker oxide layer on the surface of the sample. The $70 \times 70$ map was acquired using a step size of $0.3 \mu \mathrm{m}$, similar to the copper via samples above. A rough texture on the surface of the sample can be seen in the image. After ion processing the sample using $600 \mathrm{~V}, 5 \mathrm{~mA}$, and an angle of $4^{\circ}$ for 13 minutes, the patterns are improved as shown by the histogram in Fig. 7B. Although there is smoothing of the surface, there are larger texturing features that are seen on the surface. The oxide layer on the original surface was not a uniform coating as evidenced by the color variations across the entire surface. The non-uniform removal of the oxide layer may be responsible for the development of this texturing. In addition, there appears to be "divots" in the surface. Since this was not a long ion process, it is probable that the "divots" on the surface seen in Fig. 7B are due to abrasive particles 
that were embedded in the soft surface during polishing. Figure 7C shows the results of increasing the step size to $3.0 \mu \mathrm{m}$ which also required dynamic focusing to complete. This was done to test whether a lower magnification would give a more randomization of the crystallographic orientations of the patterns. There is little difference between the distributions shown in Figs. 7B and 7C.
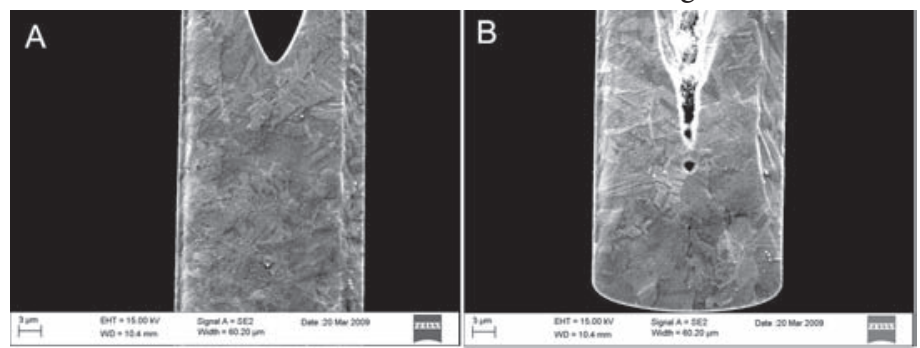

Figure 8 Copper via sample that was ion etched for 2 minutes using $600 \mathrm{~V}, 5 \mathrm{~mA}$, and an angle of $55^{\circ}$ relative to the surface normal.

The KDC-10 is also ideally suited for ion etching samples for enhancing contrast in both SEM and optical images. As shown in Fig. 3, the ion beam can treat a large sample. When the sample is tilted to a non-normal incident angle, the beam will treat an even larger area. Figure 8 shows a copper via sample that has been etched at an angle of $55^{\circ}$ relative to the surface normal for 2 minutes using $600 \mathrm{~V}$ and $5 \mathrm{~mA}$ beam current. The sample was over-etched, but it does show the potential replacing chemical etching with dry etching of larger samples such as metallurgical or ceramic materials.

The work presented here is in the early stages. We are continuing the work to evaluate the optimal conditions of using the KDC-10. Copper samples that have been ion polished at very low angles, i.e. less than $5^{\circ}$, and that have been oscillated for very long times, show an unacceptable amount of surface texture that should not occur. Since the sample is located at the eucentric height of the stage, only about half of the collimated beam is striking the sample. It is possible that sputtered material from elsewhere in the chamber is being sputtered onto the sample and is temporarily masking the sample surface and thus causing the texture. A larger sample of a soft Te alloy (approximately $2.5 \mathrm{~cm}^{2}$ ) that was ion polished at a low angle did not show any texturing. We will be testing the focusing grids to determine if the higher beam current density, which would lead to higher sputter rates and less overshoot of the beam, will improve this. We will also be putting a graphite plate behind the sample because of its low sputter yield compared to other materials. The difference in sputter yield between other materials and carbon increases with decreasing ion energy and we will be trying voltages around $200 \mathrm{~V}$ with the focusing grids to determine if that improves the surface quality.

One benefit of the ion beam that has not been mentioned above is the ability of the ion gun to clean the surface of the sample. With samples that were polished with colloidal silica and thoroughly cleaned and rinsed, contamination in the microscope was noticeable. No contamination was seen with the ion treated samples.

The authors would like to thank Clive Hayzelden of Carl Zeiss for his help and acknowledge and thank the Carl Zeiss SMT Center of Excellence at the University of California at Irvine.

\section{References}

[1] A. Barna, B. Pecz, and M. Menyhard, Ultramicroscopy. 70, (1998), p.161.

[2] A. Barna, and B. Pecz, Proc. Mat. Res. Soc., Vol. 254, (1992), p. 1.

\section{High Performance Hyperspectral Imaging Solutions for the Life Sciences}

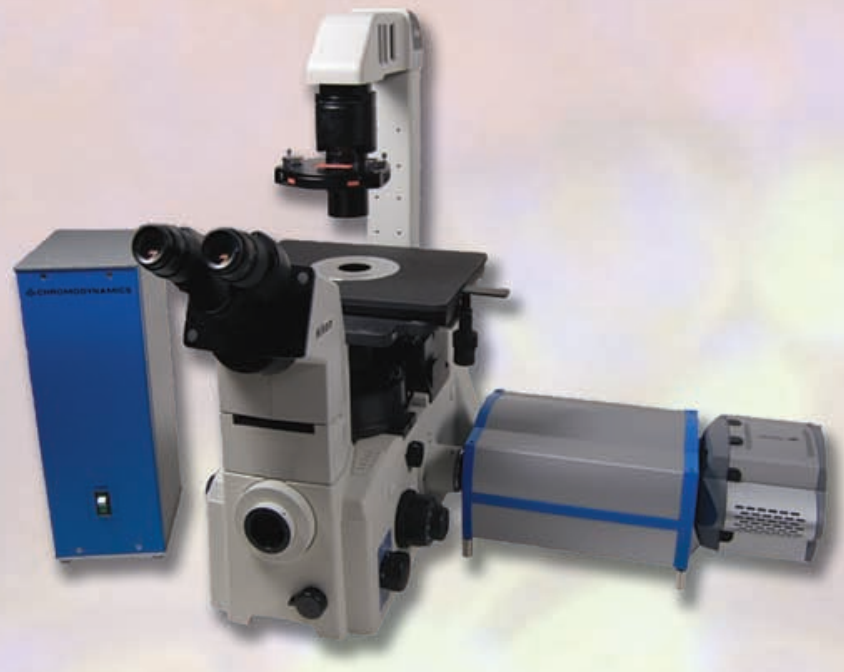

- Immunohistochemistry

- Quantum Dots (Q-dots)

- Fluorescence In-situ Hybridization (FISH)

- Fluorescence Resonance Energy Transfer (FISH)

- Spectral Karyotyping
ChromoDynamics' patented acousto-optic tuneable filter (AOTF) imaging module fulfills the promise of multispectral and hyperspectral imaging through unmatched spectral flexibility and switching speeds.

The AOTF is a high-speed, random access solid state optical filter with an adjustable optical bandpass. ChromoDynamics' proprietary AOTF technology delivers diffraction limited image quality with variable bandwidths from 1.5 to $25 \mathrm{~nm}$. Wavelength switching time, including computer control overhead, is less than 100 microseconds.

The performance capabilities of this technology make it ideally suited for high-content, high-throughput fluorescence studies, as well as spectral transmission and reflectance imaging. Potential applications range from live cell and whole animal studies to fixed slide clinical diagnostics. 